\section{Strong, Light, Multifunctional Fibers of Carbon Nanotubes with Ultrahigh Conductivity}

\author{
Natnael Behabtu, ${ }^{1}$ Colin C. Young, ${ }^{1 *}$ Dmitri E. Tsentalovich, ${ }^{1 *}$ Olga Kleinerman, ${ }^{2}$ Xuan Wang, ${ }^{1}$ \\ Anson W. K. Ma, ${ }^{1}$ E. Amram Bengio, ${ }^{1,2}$ Ron F. ter Waarbeek, ${ }^{3}$ Jorrit J. de Jong, ${ }^{3}$ \\ Ron E. Hoogerwerf, ${ }^{3}$ Steven B. Fairchild, ${ }^{4}$ John B. Ferguson, ${ }^{4}$ Benji Maruyama, ${ }^{4}$ \\ Junichiro Kono, ${ }^{1}$ Yeshayahu Talmon, ${ }^{2}$ Yachin Cohen, ${ }^{2}$ Marcin J. Otto, ${ }^{3}$ Matteo Pasquali ${ }^{1} \dagger$
}

Broader applications of carbon nanotubes to real-world problems have largely gone unfulfilled because of difficult material synthesis and laborious processing. We report high-performance multifunctional carbon nanotube (CNT) fibers that combine the specific strength, stiffness, and thermal conductivity of carbon fibers with the specific electrical conductivity of metals. These fibers consist of bulk-grown CNTs and are produced by high-throughput wet spinning, the same process used to produce high-performance industrial fibers. These scalable CNT fibers are positioned for high-value applications, such as aerospace electronics and field emission, and can evolve into engineered materials with broad long-term impact, from consumer electronics to long-range power transmission.

$\mathrm{O}$ the molecular level, carbon nanotubes (CNTs) have an outstanding combination of mechanical strength and stiffness, electrical and thermal conductivity, and low density, making them ideal multifunctional materials that combine the best properties of polymers, carbon fibers, and metals (1). However, such outstanding properties have remained elusive on a macroscopic scale. Handling CNTs with sufficient length, stiffness, and chemical inertness introduces major challenges in material processing. Here we report lightweight fibers that approach the high specific strength of polymeric and carbon fibers, while also achieving the high specific electrical conductivity of metals and the specific thermal conductivity of graphite fibers.

Two distinct routes have been developed for manufacturing neat CNT fibers (2). One route employs a solid-state process wherein CNTs are either directly spun into a fiber from the synthesis reaction zone $(3,4)$ or from a CNT forest grown on a solid substrate (5). This approach does not lend itself to the typical easy scale-up of chemical processes, as it combines multiple steps into a single one, limiting the options for process and material optimization. Indeed, solidstate fibers have low packing and poor orien-

${ }^{1}$ Department of Chemical and Biomolecular Engineering, Department of Chemistry, Department of Electrical and Computer Engineering, Department of Physics and Astronomy, Applied Physics Program, The Smalley Institute for Nanoscale Science and Technology, Rice University, Houston, TX 77005, USA. ${ }^{2}$ Department of Chemical Engineering, Technion-Israel Institute of Technology and Russell Berrie Nanotechnology Institute (RBNI), Haifa 32000, Israel. ${ }^{3}$ Teijin Aramid, Arnhem, Netherlands. ${ }^{4}$ Materials and Manufacturing Directorate, Air Force Research Laboratory, Wright-Patterson Air Force Base, $\mathrm{OH}$ 45433, USA.

*These authors contributed equally to this work. †To whom correspondence should be addressed. E-mail: mp@rice.edu tation, and include impurities within their structure (6). Despite these shortcomings, solid-state CNT fibers have delivered the best properties so far $(3,4,7-9)$. The reason for this relative success is the length of the CNTs that constitute these fibers $-1 \mathrm{~mm}$ or more (2). Longer CNTs reduce the number of $\mathrm{CNT}$ ends in a fiber, yielding greater strength (10) and reducing CNT junctions, which increases electrical and thermal conductivity (11). The alternate fiber production route - wet spinning - was the first method for producing CNT fibers (12). In this process, premade CNTs are dissolved or dispersed in a fluid, extruded out of a spinneret, and coagulated into a solid fiber by extracting the dispersant. Wet spinning is easily scaled to industrial levels and is indeed the route by which highperformance fibers are manufactured (including ballistic fibers such as Kevlar and Twaron and structural fibers such as Toho Tenax and Thornel carbon fibers) (13). Decoupling the synthesis of CNTs from the spinning of the fibers allows the independent optimization of the two steps and enables CNT purification. An important variation of the original wet spinning method was the use of acid as a solvent and standard coagulants like water (14), thereby simplifying the original method (12) by avoiding ing polymer from the coagulation bath. So far, wet spinning from acid has yielded the most highly ordered and dense CNT fibers (14). Yet, their properties have been disappointing. Inadequate CNT length $[\sim 0.5 \mu \mathrm{m}(14)]$ has been the presumed culprit (2), and the literature concurs that wet spinning is inappropriate for handling long CNTs. Here we show that exciting properties can be achieved by wet-spinning $\sim 5-\mu \mathrm{m}$ "short" CNTs into high-performance multifunctional fibers.

High-quality CNTs (figs. S1 to S6) were dissolved (15) in chlorosulfonic acid (the only surfactants in the CNT dispersion and eliminat- known CNT solvent) (16-18) at a concentration of 2 to 6 weight (wt) $\%$ and filtered to remove particles, in order to form a spinnable liquid crystal dope (Fig. 1A and fig. S7). The dope was extruded through a spinneret (65- to $130-\mu \mathrm{m}$ diameter) into a coagulant (acetone or water) to remove the acid. The forming filament was collected onto a winding drum (Fig. 1, B to E, and movie S1). The linear velocity of the drum was higher than the dope speed at the spinneret exit, to ensure high CNT alignment by continuous stretching and tensioning of the filament. The fibers were further washed in water and dried in an oven at $115^{\circ} \mathrm{C}$.

Fibers were tested for mechanical, electrical, and thermal properties. Tensile strength, modulus, and elongation to break (15) were determined from tensile break tests on macroscopic $(\sim 20 \mathrm{~mm}$ long) individual filaments cut from large spools ( $\sim 100$ to $500 \mathrm{~m}$ ). Stress was calculated by dividing the applied force by the fiber cross-sectional area determined by scanning electron microscopy (SEM) (fig. S8) and light microscopy (fig. S9). The average tensile strength was $1.0 \pm 0.2 \mathrm{GPa}$ (best value $1.3 \mathrm{GPa}$, fig. S10A), and the average modulus was $120 \pm 50 \mathrm{GPa}$ (best value $200 \mathrm{GPa}$; fig. S10, B and C). The average elongation at break for these fibers was $1.4 \pm 0.5 \%$. These same fibers displayed high electrical conductivity [measured by two- and four-point probe on 25-mm single filaments (15)], on average $2.9 \pm$ $0.3 \mathrm{MS} / \mathrm{m}$ (resistivity of $35 \pm 3$ microhm $\mathrm{cm}$ ) at room temperature; doping by iodine (15)-a known, stable CNT dopant (19)-increased conductivity to $5 \pm 0.5 \mathrm{MS} / \mathrm{m}$ (resistivity $22 \pm 4$ microhm cm, best value of 17.5 microhm cm); these values were stable over 1 year in laboratory conditions and also under thermal cycling to $200^{\circ} \mathrm{C}$ in air for 24 hours. We also measured an average thermal conductivity of $380 \pm$ $15 \mathrm{~W} / \mathrm{m} \mathrm{K}$ on $\sim 1.5$-mm-long samples using the 3 -omega method $(15,20)$. Iodine doping doubled thermal conductivity $(635 \mathrm{~W} / \mathrm{m} \mathrm{K})$. Such high thermal conductivity remains unchanged after annealing at $600^{\circ} \mathrm{C}$, whereas the electrical conductivity drops by an order of magnitude to $0.4 \mathrm{MS} / \mathrm{m}$ (resistivity of $240 \mathrm{microhm} \mathrm{cm}$ ). Fiber density [measured by weighing a $60-\mathrm{m}$ filament with $(9.0 \pm 0.6)-\mu$ m diameter] was $1.3 \pm$ $0.1 \mathrm{~g} / \mathrm{cm}^{3}$; iodine doping increased it to $1.4 \mathrm{~g} / \mathrm{cm}^{3}$, on the basis of a $10 \%$ mass increase determined from thermal gravimetric analysis (fig. S11).

These combined properties are remarkable when compared to those of other CNT fibers and high-performance materials (Fig. 2 and table S2); these properties are related to CNT length, alignment, type (including diameter and number of walls), graphitic character, and purity. Tensile strength shows a 10 -fold improvement over wet-spun fibers of $0.5-\mu \mathrm{m}$ CNTs $[\sim 0.11 \mathrm{GPa}(14)]$ and is comparable to the best macroscopic samples of solid-state spun fibers of millimeter-long CNTs [best values of $\sim 1.8 \mathrm{GPa}$ assuming a density of $\left.1 \mathrm{~g} / \mathrm{cm}^{3}(7)\right]$. We find that $\mathrm{CNT}$ length, aspect ratio, and purity 
are key to strength improvements, because of better CNT-CNT stress transfer and lower defect density; in contrast, CNT orientation, graphitic character, and type are not as critical for attaining high strength, although a higher number of walls lowers the specific strength. The modulus is improved over that of earlier wetspun $0.5-\mu \mathrm{m}$ CNT fibers $[\sim 120 \mathrm{GPa}(14)]$ and higher than that of the best solid-state CNT fibers $\left[\sim 120 \mathrm{GPa}\right.$ assuming a density of $1 \mathrm{~g} / \mathrm{cm}^{3}$ (7)], but lower than the modulus of graphite fibers (GF) $(\sim 1 \mathrm{TPa})$. Modulus is principally affected by orientation, which was already high in earlier studies (14); the increased CNT orientation in our fibers is counterbalanced by the lowered theoretical modulus $(\sim 350 \mathrm{GPa})$ due to larger CNT diameter (21). Electrical conductivity of doped fibers is 10 -fold that of wet-spun $0.5-\mu \mathrm{m}$ CNT fibers and the best continuous, undoped solid-state fibers $[0.5 \mathrm{MS} / \mathrm{m}(14,22)]$; three to five times better than that of the best doped CNT fibers $[\sim 1.3 \mathrm{MS} / \mathrm{m}(23)]$ and isolated ropes $[\sim 1 \mathrm{MS} / \mathrm{m}(24,25)]$; and comparable to that of the best solid-state, acid-densified, iodine-doped individual CNT fibrils [5.8 MS/m (26)]. The fiber electrical conductivity did not degrade when kinks were progressively introduced (figs. S12 and S13), indicating that these fibers, unlike copper (27), resist bending fatigue. We find that electrical conductivity is not as sensitive as modulus to CNT alignment and impurities; length is not critical in the tested range of $\sim 3$ to $\sim 7 \mu \mathrm{m}$ (but is likely important in the range 0.5 to $3 \mu \mathrm{m}$ ). The key improvements over earlier fibers are due to the combined effect of CNT length, type, and graphitic character, as also observed recently in CNT films (28). Thermal conductivity appears to follow electrical conductivity [as in conventional carbon fibers (29)]; it is $\sim 30$ times as high as that of wet-spun $0.5-\mu \mathrm{m}$ CNT fibers $[21 \mathrm{~W} / \mathrm{m} \mathrm{K}(14)], \sim 10$ times as high as that of the best solid-state CNT fibers [60 W/m K (30)], and $\sim 3$ times as high as that of the best magnetically aligned discrete CNT films $[200 \mathrm{~W} / \mathrm{m} \mathrm{K}(31)]$.

Figure 2, B and C, compare CNT fibers (from this article and from the literature) to the materials with the best mechanical, electrical, and thermal properties. Our CNT fibers combine the typical specific electrical conductivity of metal wires (copper, silver, and aluminum) with the typical specific strength of high-performance carbon fibers.

To understand the fiber structure-properties relationship, we studied fiber morphology [by highresolution SEM (HR-SEM) (15)], alignment [by single filament wide angle $\mathrm{x}$-ray diffraction, WAXD (15)], and packing fraction [by high-resolution
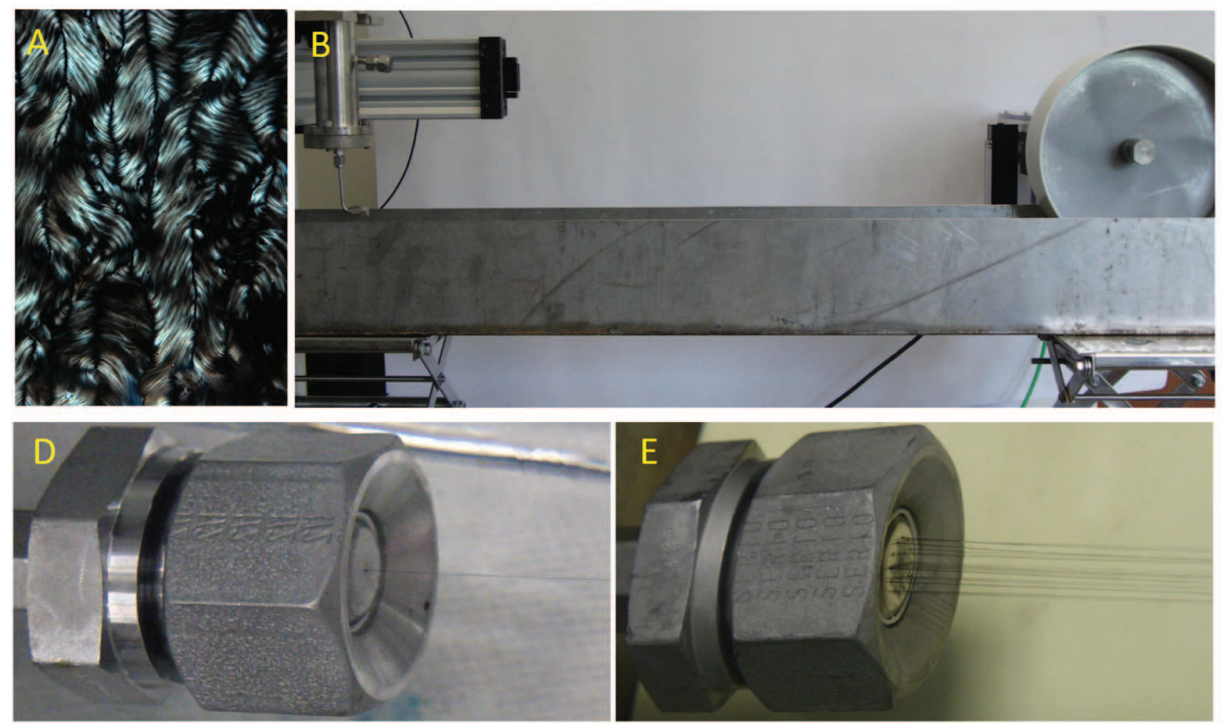

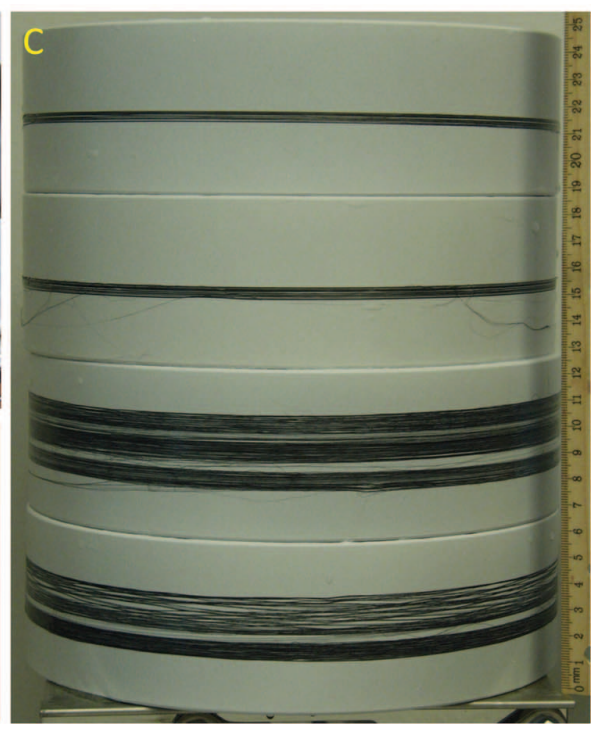

tion bath; the fiber is continuously collected on the winding drum (right). (C) Winding drums with collected fibers (100 to $500 \mathrm{~m}$ on each drum). (D and E) Close-up view of a single- and 19- filament spinning.
CNT in chlorosulfonic acid). (B) Fiber spinning set-up. The fluid is extruded from the spinning chamber (left) through a spinneret immersed in a coagula-
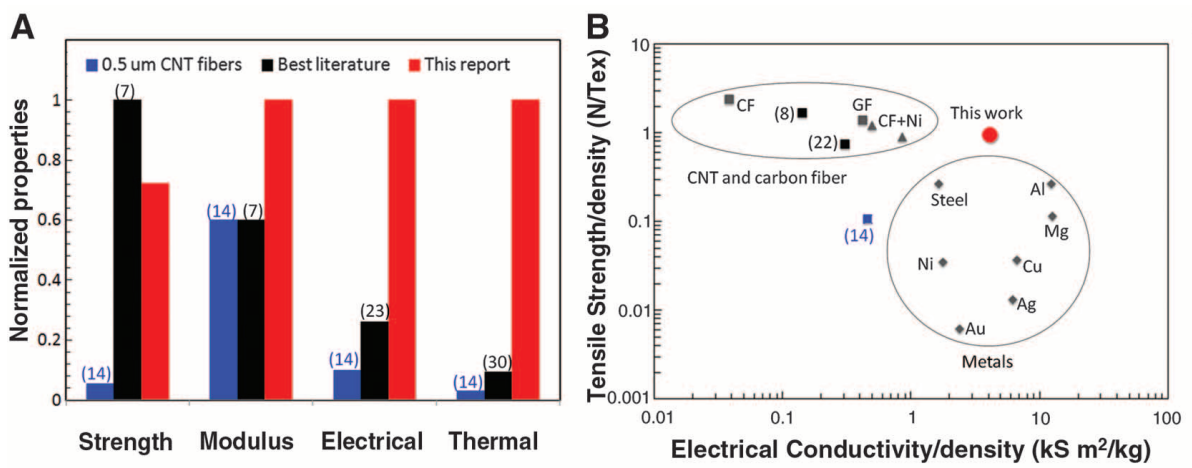

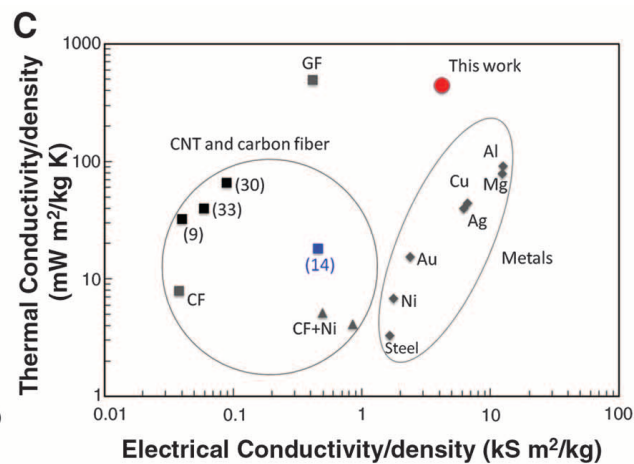

Electrical Conductivity/density ( $\mathrm{kS} \mathrm{m}^{2} / \mathrm{kg}$ )
Fig. 2. Properties of continuous, neat CNT fibers. Black denotes literature values $(7,8,9,22,23,30,33)$, blue denotes earlier wet-spun 0.5- $\mu \mathrm{m}$ CNT fibers (14), and red denotes fibers in this report. (A) Comparison of properties normalized to the highest value. (B) Ashby plots of specific tensile strength versus specific electrical conductivity of metals (gray diamonds), pitch (GF) and PAN (CF) carbon fibers (gray squares), nickel-coated CF (gray triangles), CNT fibers (black and blue squares), and CNT fibers from this report (red circle). Carbon and CNT fibers fall in a high-strength, low-conductivity region; metals define a high-conductivity, low-strength region. (C) Specific electrical conductivity versus specific thermal conductivity Ashby plot, showing distinct regions for metals and carbon fiber. 
transmission electron microscopy (TEM)]. HRSEM revealed that the fiber consists of wellaligned, thin CNT fibrils (Fig. 3, A and B) (typical diameter of 10 to $100 \mathrm{~nm}$ and length $>50 \mu \mathrm{m}$ ), similar to wet-spun $0.5-\mu \mathrm{m}$ CNT fibers (14), as well as high-strength polymeric fibers (32). WAXD showed sharp reflections at $2 \theta=25.3^{\circ}$ (Fig. 3C and fig. S14), due to planes perpendicular to the nanotube axis (15). The azimuthal scan of this peak gave a full width at half maximum (FWHM) of $9.4^{\circ}$ (which corresponds to a Herman orientation factor of 0.986 ), averaged over four different points along a single fiber (lowest and highest values were $7.3^{\circ}$ and $11.2^{\circ}$ ). Electron diffraction on isolated single fibrils (peeled off the fibers) displayed better order $\left(\mathrm{FWHM}=5^{\circ}\right)$, indicating slight misalignment of fibrils within the fiber (Fig. $3 \mathrm{C}$ ). This compares to $\mathrm{FWHM}=31^{\circ}$ for wet-spun $0.5-\mu \mathrm{m}$ CNT fibers (14) and FWHM between $10^{\circ}$ and $14^{\circ}$ for solid-state spun fibers (33). We also quantified packing fraction by calculating a maximum theoretical packing density of $1.5 \mathrm{~g} / \mathrm{cm}^{3}$ from high-resolution TEM on the starting CNT material (15). Hence, the fiber density $\left(1.3 \mathrm{~g} / \mathrm{cm}^{3}\right)$ is $\sim 90 \%$ of the theoretical close-packed density. We verified the high packing fraction by visualizing the fiber cross section. The images showed occasional $\sim 100$-nm voids, but no larger voids (Fig. 3, D and E). This packing density for neat carbon nanotube fibers compares with $78 \%$ for wet-spun $0.5-\mu \mathrm{m}$ CNT fibers (14) and $\sim 50 \%$ for solid-state fibers (3).
To understand the relative effect of CNT alignment, packing, and doping on the conduction mechanism in these fibers, we studied the temperature-dependent conductivity of annealed fibers and isotropic films, as well as acid-doped and iodine-doped fibers (differing in extent of doping). All samples show two regimes (Fig. 4A): At low temperature the conductivity rises with temperature (semiconducting behavior), whereas at high temperature the conductivity drops (metallic behavior). The crossover temperature (indicated by an arrow) is highest in the annealed fiber and film and lowest in the iodine-doped fiber. The low-temperature behavior can be understood in terms of carrier hopping or tunneling, i.e., inter-CNT transport, which can be substantially affected by mismatch of CNT types (metallic versus semiconducting) and degree of alignment. The high-temperature, metallic behavior reflects diffusive, intra-CNT transport, for which the conductivity is limited by electron-phonon scattering. Each temperature-dependent curve can be well fitted (15) by an equation (table S2) that combines the two regimes (34), as shown in Fig. 4A. The annealed fiber is $\sim 3.5$ times more conductive than the annealed film. This is due to the CNT alignment in the fiber, which increases CNT density and overlap and decreases their spacing, facilitating inter-CNT transport. Doping the fiber by residual acid or iodine further improves the conductivity by $\sim 5$ - to 10 -fold because doping increases the intra-CNT conductivity of the semi- conducting CNTs and also increases disorder that helps relax the momentum conservation required for inter-CNT transport (35). These advantages of doping seem to prevail over the conductivity loss due to enhanced electron-impurity scattering

Above $\sim 1 \mathrm{~K}$, the thermal conductivity is dominated by phonon transport and hence not closely correlated to the electrical conductivity (Fig. 4B). We observe in a macroscopic sample a crossover (at 250 to $300 \mathrm{~K}$ ) from impurity-phonon scattering to three-phonon Umklapp scattering, similar to the behavior of a single multiwalled carbon nanotube (MWNT) (36). The conductivity is about $20 \%$ of the single-MWNT value, possibly due to quenching by the inter-CNT coupling, but 10 to 100 times that of other macroscopic CNT samples, usually limited by weak inter-CNT transport due to misalignment. Acid removal by annealing roughly doubles the fiber thermal conductivity, presumably due to reduced phononimpurity scattering; surprisingly, annealing in the presence of iodine yields comparable improvement (15), showing that both high electrical and thermal conductivity can be attained on the same fiber.

We demonstrate the multifunctional properties of our fibers by supporting and wiring a light-emitting diode (LED, $46 \mathrm{~g}$ ) and by fabricating cold electron-emitting cathodes. The LED was supported by two CNT fibers [(24 \pm 1$)-\mu \mathrm{m}$ diameter, Fig. 4C]. The stress in each fiber was
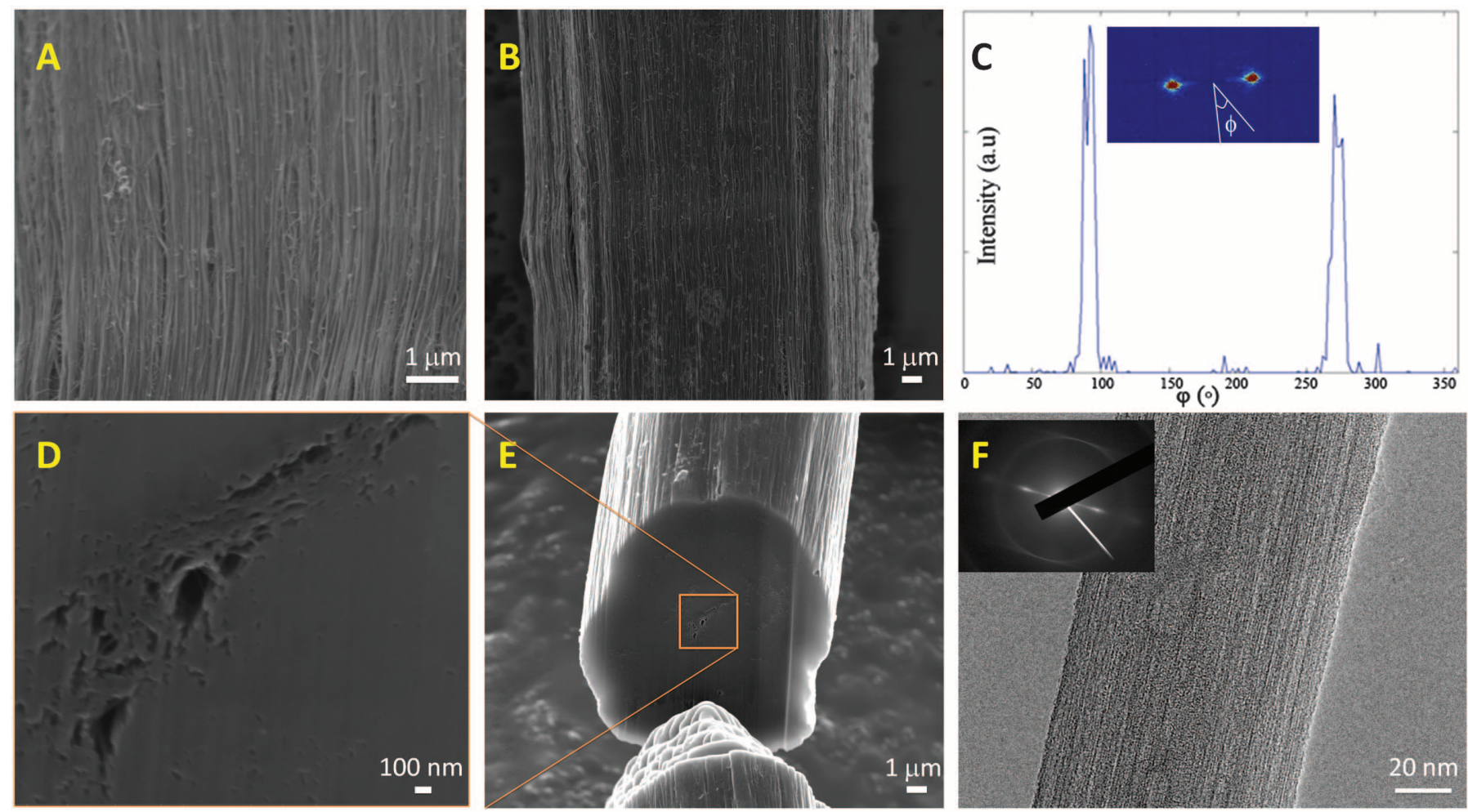

Fig. 3. (A) High- and (B) low-magnification SEM showing the typical morphology of CNT fibers composed of 100-nm-thick fibrils aligned along the fiber axis. (C) Single-fiber x-ray diffraction (inset) and azimuthal scan showing the high fiber alignment. (D and E) SEM images showing fiber's cross section after cutting by focused ion beam. There are no micrometer-sized voids and few hundred-nanometer-sized voids. (F) Single-fibril TEM micrograph and electron diffraction (inset) showing near-crystalline packing within a fibril. 
$\sim 500 \mathrm{MPa}$, well above the breaking strength of copper wires, but the filament resistance was low enough to run $30-\mathrm{mA}$ current $\left(6.6 \times 10^{3} \mathrm{~A} / \mathrm{cm}^{2}\right.$ current density) and light the device.

A field-emitting device (15) was fabricated in a cathode (fiber)-anode configuration (Fig. 4D). The emitted current density was $5.8 \times 10^{3}$ $\mathrm{A} / \mathrm{cm}^{2}$ (3.6 mA from a 9- $\mu \mathrm{m}$-diameter fiber) at $0.86 \mathrm{~V} / \mu \mathrm{m}$ and $1-\mathrm{mm}$ anode-cathode distance (Fig. 4E). This current density is more than two orders of magnitude higher than that of wetspun $0.5 \mu \mathrm{m}$ CNT fibers (14), graphite fibers (a common material for large area cathodes), and typical solid-state spun CNT fibers (37-41). The high current density of our fibers is due to the high electrical and thermal conductivity, which reduces the fiber temperature by generating less Joule heating and by dissipating the heat efficiently, preventing tip failure.

High performance is only one of the two requisites for technology adaptation. Ease of manufacturing is key for cost control through process scale-up (15). We demonstrated scalability of solution spinning by using a multihole spinneret under the same conditions as for the single hole spinning — same linear extrusion rate $(\sim 10 \mathrm{~m} / \mathrm{min})$ per hole and spinneret diameter (Fig. 1E). The only required change was a simple switch of the spinning plate, with no alteration of any other hardware. The produced 19-filament fibers showed properties comparable to those of the monofilament ones.

The combined achievement of CNT fiber multifunctionality and a scalable manufacturing process is a major step toward macroscopic CNT-based materials. As with high-performance polymeric fibers, optimal morphology (alignment, packing density, lack of impurities, and high-quality molecular constituents) is crucial for the final CNT fiber properties. Yet, the fiber properties are still a fraction ( $\sim 2$ to $20 \%$ ) of the ultimate intrinsic properties of individual CNTs and the expected properties of their fibers. Increasing fiber strength will require the production of longer, thin, defect-free CNTs (speculatively, length $>50 \mu \mathrm{m}$, diameter $<3 \mathrm{~nm}$ ), and preferably single-walled carbon nanotubes (SWNTs). Reaching ultimate conductivity may require the synthesis of all-armchair SWNTs. The consistent synthesis of large quantities (gram amounts or higher)
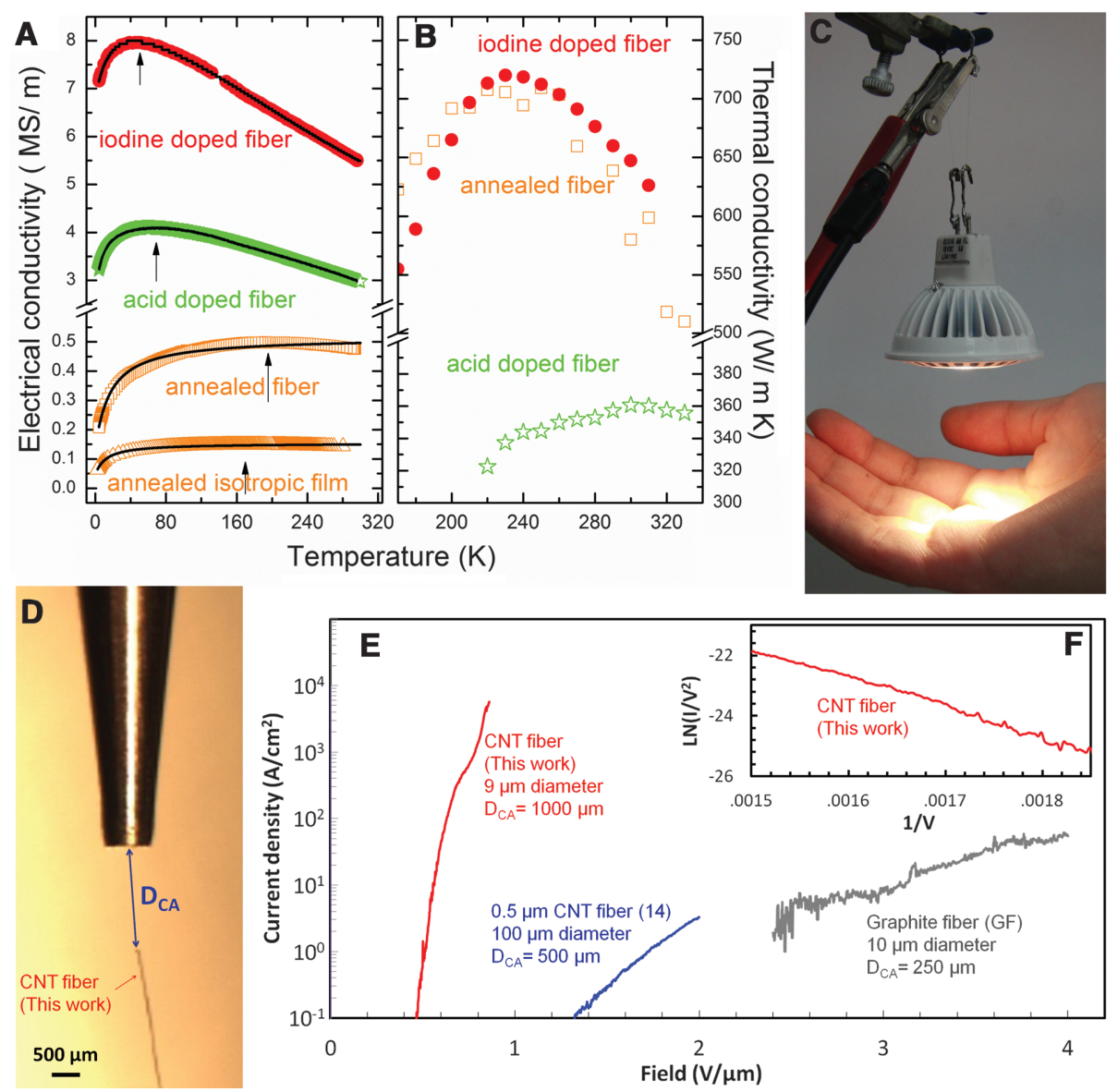

Fig. 4. (A) Temperature dependence of electrical and (B) thermal conductivity of acid-doped, annealed, and iodine-doped fibers and an annealed random film. (C) A 46-g light-emitting diode lit and suspended by two $24-\mu \mathrm{m}$-thick CNT fibers. (D) Field emission set-up, with a single-fiber cathode facing the anode. (E) Current density versus field strength of GF (gray), wet-spun $0.5-\mu \mathrm{m}$ CNT fiber (blue) and our CNT fiber (red). (F) The I-V data in simplified Fowler-Nordheim coordinates fall on a straight line, indicating metallic field emission.

of defect-free CNTs with such control on length, diameter, and chirality is a key technological barrier that must be overcome to enlarge greatly the design space of CNT fibers and to make highperformance macroscopic materials of CNTs.

\section{References and Notes}

1. R. H. Baughman, Science 290, 1310 (2000).

2. N. Behabtu, M. J. Green, M. Pasquali, Nano Today 3, 24 (2008).

3. K. K. Koziol et al., Science 318, 1892 (2007).

4. S. D. Lashmore, J. J. Brown, K. J. Chaffee, B. Resnicoff, P. Antoinette, U.S. patent 7993620 (2011).

5. M. Zhang, K. R. Atkinson, R. H. Baughman, Science 306, 1358 (2004).

6. R. J. Davies, C. Riekel, K. K. Koziol, ]. J. Vilatela, A. H. Windle, J. Appl. Cryst. 42, 1122 (2009).

7. S. Boncel, R. M. Sundaram, A. H. Windle, K. K. Koziol, ACS Nano 5, 9339 (2011).

8. K. Liu et al., Nanotechnology 21, 045708 (2010).

9. A. E. Aliev et al., Carbon 45, 2880 (2007).

10. B. I. Yakobson, G. Samsonidze, G. G. Samsonidze, Carbon 38, 1675 (2000).

11. A. Nieuwoudt, Y. Massoud, IEEE Trans. Electron. Dev. 55, 2097 (2008).

12. B. Vigolo et al., Science 290, 1331 (2000).

13. H. H. Yang, Aromatic High-Strength Fibers (Wiley, New York, 1989).

14. L. M. Ericson et al., Science 305, 1447 (2004).

15. Supplementary materials are available on Science Online.

16. S. Ramesh et al., J. Phys. Chem. B 108, 8794 (2004).

17. V. A. Davis et al., Nat. Nanotechnol. 4, 830 (2009).

18. A. N. G. Parra-Vasquez et al., ACS Nano 4, 3969 (2010).

19. L. Grigorian et al., Phys. Rev. Lett. 80, 5560 (1998).

20. L. Lu, W. Yi, D. L. Zhang, Rev. Sci. Instrum. 72, 2996 (2001).

21. T. Yamamoto, K. Watanabe, E. Hernández, in Carbon Nanotubes: Advanced Topics in the Synthesis, Structure, Properties and Applications (Springer/Heidelberg, Berlin, 2008), pp. 165-194.

22. X. H. Zhong et al., Adv. Mater. 22, 692 (2010).

23. J. Alvarenga et al., Appl. Phys. Lett. 97, 182106 (2010).

24. J. E. Fischer et al., Phys. Rev. B 55, R4921 (1997).

25. A. Thess et al., Science 273, 483 (1996).

26. Y. Zhao, J. Wei, R. Vajtai, P. M. Ajayan, E. V. Barrera, Sci. Rep. 1, 83 (2011)

27. P. Jarosz et al., Nanoscale 3, 4542 (2011).

28. F. Mirri et al., ACS Nano 6, 9737 (2012).

29. J. Heremans, I. Rahim, M. S. Dresselhaus, Phys. Rev. $B$ 32, 6742 (1985).

30. M. B. Jakubinek et al., Carbon 50, 244 (2012).

31. J. Hone et al., Appl. Phys. Lett. 77, 666 (2000).

32. Y. Cohen, E. L. Thomas, Polym. Eng. Sci. 25, 1093 (1985).

33. J. J. Vilatela, R. Khare, A. H. Windle, Carbon 50, 1227 (2012).

34. A. B. Kaiser, Rep. Prog. Phys. 64, 1 (2001).

35. A. A. Maarouf, C. L. Kane, E. J. Mele, Phys. Rev. B 61, 11156 (2000).

36. P. Kim, L. Shi, A. Majumdar, P. L. McEuen, Phys. Rev. Lett. 87, 215502 (2001).

37. L. Ci et al., Adv. Mater. 19, 1719 (2007).

38. Y. Yang, L. Liu, S. Fan, Vacuum 86, 885 (2012).

39. H.-S. Jang, S. K. Jeon, S. H. Nahm, Carbon 48, 4019 (2010).

40. A. A. Zakhidov et al., Appl. Phys. A Mater. Sci. Process. 88, 593 (2007).

41. The highest emission reported for solid-state spun CNT fibers is $1500 \mathrm{~A} / \mathrm{cm}^{2}$ (1.2 mA from $10-\mu \mathrm{m}$-diameter fiber), but this value is somewhat surprising because of the relatively low electrical $(60 \mathrm{kS} / \mathrm{m})$ and thermal $(<100 \mathrm{~W} / \mathrm{m} \mathrm{K})$ conductivity of these solid-state CNT fibers (40).

Acknowledgments: We thank F. Mirri for the CNT film; T. Back, D. Ramsamoedj, E. Klop, E. Steyn, T. Cohen-Hymas, P. M. Ajayan, B. Carey, F. Matteini, A. Mizes, V. Parolkar, A. Choi, and S. Odumabo for help with fiber characterization; and K. McElrath, E. Vega, X. Hu, W. Adams, R. Hauge, and 
E. Thomas for useful discussions. This work was supported by Teijin Aramid BV, Teijin Techno Products Ltd., Air Force Office of Scientific Research FA9550-09-1-0590, Russell Berrie Nanotechnology Institute, Air Force Research Laboratory FA8650-07-2-5061, the U.S. Department of Defense National Defense Science and Engineering Graduate fellowship, and the Welch Foundation (C-1668 and Evans Attwell fellowship).
European provisional patent application EP12177741 was filed 25 July 2012

\section{Supplementary Materials}

www.sciencemag.org/cgi/content/full/339/6116/182/DC1

Materials and Methods

Supplementary Text
Figs. $\mathrm{S} 1$ to $\mathrm{S} 3$

Tables S1 to S3

References (42-52)

27 July 2012; accepted 21 November 2012 10.1126/science.1228061

\section{Bio-Inspired Polymer Composite Actuator and Generator Driven by Water Gradients}

\author{
Mingming Ma, ${ }^{1}$ Liang Guo, ${ }^{1}$ Daniel G. Anderson, ${ }^{1,2}$ Robert Langer ${ }^{1,2 *}$
}

Here we describe the development of a water-responsive polymer film. Combining both a rigid matrix (polypyrrole) and a dynamic network (polyol-borate), strong and flexible polymer films were developed that can exchange water with the environment to induce film expansion and contraction, resulting in rapid and continuous locomotion. The film actuator can generate contractile stress up to 27 megapascals, lift objects 380 times heavier than itself, and transport cargo 10 times heavier than itself. We have assembled a generator by associating this actuator with a piezoelectric element. Driven by water gradients, this generator outputs alternating electricity at $\sim 0.3$ hertz, with a peak voltage of $\sim 1.0$ volt. The electrical energy is stored in capacitors that could power micro- and nanoelectronic devices.

$\mathrm{P}$ olymeric materials that reversibly change shape, size, or mechanical properties in response to external stimuli have attracted considerable interest because of their potential applications as actuators for biomedical and me- chanical purposes (1). Based on their energy sources for actuation, responsive polymeric materials can be divided into three classes: electroactive polymers $(2,3)$, light- or thermal-responsive elastomers (4-9), and $\mathrm{pH}$ - or solvent-responsive gels (10-14). Many organisms use water-sorptioninduced swelling for actuation (15). Several types of water-responsive hydrogels have been developed for actuator fabrication (10), but they exhibit slower response, lower stress generation, and marginal stability than do animal muscle fibers.

Polypyrrole (PPy) is an electroactive polymer with many desirable properties that could allow it to act as an artificial muscle $(16,17)$. PPy can also absorb water and change its shape, which allows it to drive motion in a rotary actuator (18). However, existing PPy rotary actuators only weakly output mechanical force or power, in contrast to PPy-based electroactuators $(16,18)$. Inspired by the network structure of animal dermis, in which rigid collagen fibers reinforce an elastic

${ }^{1}$ David H. Koch Institute for Integrative Cancer Research, Massachusetts Institute of Technology (MIT), Cambridge, MA 02139, USA. ${ }^{2}$ Harvard-MIT Division of Health Sciences and Technology, and Department of Chemical Engineering, MIT, Cambridge, MA 02139, USA.

${ }^{\star}$ To whom correspondence should be addressed. E-mail: rlanger@mit.edu
Fig. 1. Characterization of PEEPPy composite films. (A) A PEEPPy composite film (black) is composed of PPy polymer chains (gray lines) and a PEE-borate network (red lines). The structure changes (involving $\mathrm{H}$ bonds and borate ester bonds) in response to water (blue dots) sorption and desorption. (B) PEE-PPy weight change (red) synchronizes with air humidity change (black). (C) ATR-IR spectra showing $H / D$ exchange between the PEE-PPy film and water vapor. Top to bottom: before $\mathrm{D}_{2} \mathrm{O}$ exposure and $0,1,2,3$, and 4 min after $\mathrm{D}_{2} \mathrm{O}$ exposure. Dashed lines indicate the three pairs of shifting peaks. (D) A PEE-PPy film maintains its flexibility and mirrorlike surface after 6 months of open storage.
A

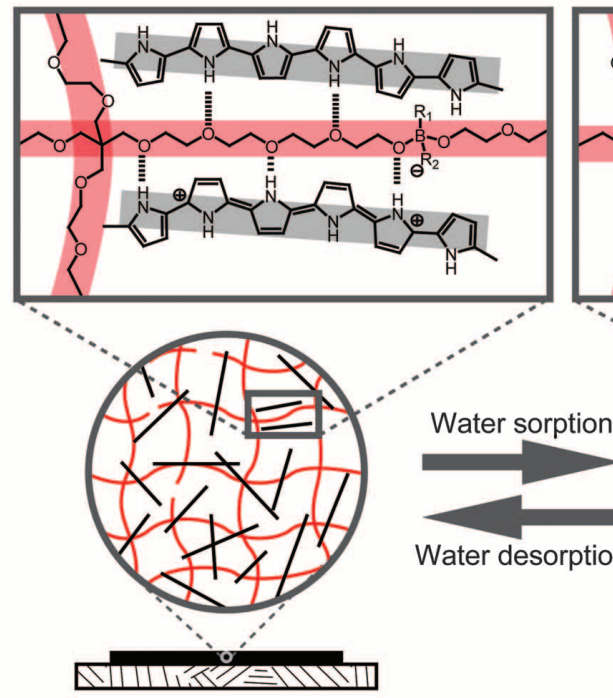

B

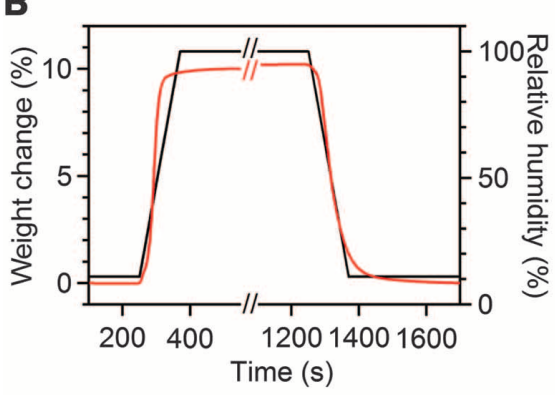

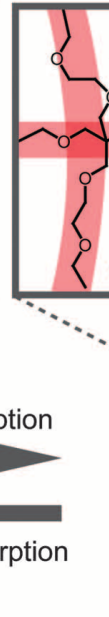

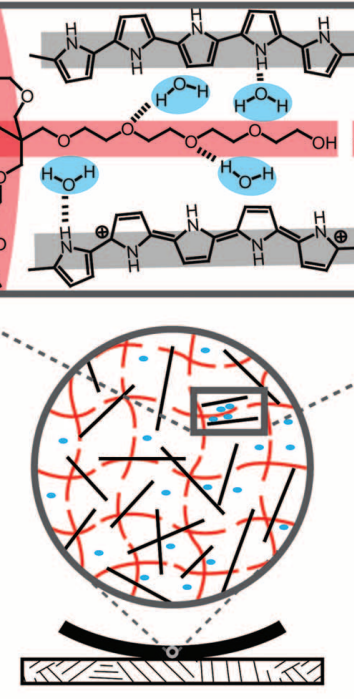

C

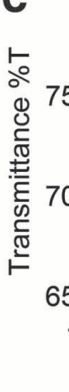

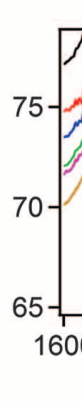

$\bar{i}=$ Hydrogen bond $\mathrm{R}_{1}, \mathrm{R}_{2}=\mathrm{F}, \mathrm{OH}$ or OR 